\title{
Multichannel fiber Bragg grating for temperature field monitoring
}

\author{
W. ZHANG, ${ }^{1,3}$ A. GBADEBo, ${ }^{2,3}$ Y. SUn, ${ }^{1}$ E. G. TURITSYNA, ${ }^{2}$ J. A. R. \\ WILLIAMS, ${ }^{2}$ Q. SUN, ${ }^{1}$ Z. YAN, ${ }^{1,2,{ }^{*}}$ D. LIU, ${ }^{1}$ AND L. ZHANG ${ }^{2}$ \\ ${ }^{I}$ School of Optical and Electronic Information, National Engineering Laboratory for Next Generation \\ Internet Access System, Huazhong University of Science and Technology, Wuhan, China \\ ${ }^{2}$ Aston Institute of Photonic Technologies, Aston University, Aston Triangle, Birmingham B4 7ET, UK \\ ${ }^{3}$ These authors contributed equally to this article \\ yanzhijun@gmail.com
}

\begin{abstract}
We demonstrate a multichannel fiber Bragg grating (MC-FBG) based distributed temperature field sensor with millimeter-order spatial resolution. The MC-FBG was designed by using the layer peeling (LP) algorithm with a tailored group delay characteristic and fabricated using seamless UV-inscription. We have achieved a 21-channel MC-FBG with 0.2 $\mathrm{nm}$ bandwidth of each channel and $0.5 \mathrm{~nm}$ channel gap. The sensor was tested by using a temperature field distribution. Experimental results show that the sensor had a spatial resolution of $3 \mathrm{~mm}$ and could measure a maximum temperature gradient of $7.85^{\circ} \mathrm{C} / \mathrm{mm}$.
\end{abstract}

(C) 2019 Optical Society of America under the terms of the OSA Open Access Publishing Agreement

\section{Introduction}

Temperature field monitoring is commonly required in the chemical industry [1], for heat management in microprocessors [2], and in wearable devices [3]. The temperature gradient contains information about the working state of these devices and can be utilized to ensure optimal operation. In order to precisely map the physical parameters and gradients, the spatial resolution of the temperature sensor has to be as high as possible. There are two main temperature field mapping methods in use which can achieve a spatial resolution of less than a millimeter: thermometry based on nuclear magnetic resonance (NMR) [1] and electron backscatter diffraction [4]. However, they are not suitable for in situ measurement in many applications due to their large size and electromagnetic interference.

Distributed optical fiber sensors, as an alternative solution for measuring temperature gradients, have the advantages of small size, a linear temperature response, low electromagnetic interference and ease of integration [5-7]. Traditional distributed optical fiber sensors are based on Rayleigh [8,9], Raman [10,11], or Brillouin [12,13] scattering processes. The spatial resolution of these distributed sensors is primarily determined by either the bandwidth or pulse width of the interrogating source signal which is typically a trade-off with the power of the detected signal [14]. Due to very weak back scattering signal, massive averaging and signal processing are usually required to provide sufficient signal-to-noise ratio (SNR). This significantly increases the demodulation time and the complexity and cost of the interrogation systems.

A fiber Bragg grating (FBG) structure is an artificial scattering unit which produces a strong, directional back scattered signal. For distributed sensing applications, the most widely used scheme is a FBG array sensor using wavelength division multiplexing $[15,16]$. However, it is difficult to reach millimeter-order spatial resolution with this approach. Recently, researchers have achieved real time distributed sensing with a spatial resolution of several millimeters by combining a high density FBG array with optical frequency domain reflectometry (OFDR) [17-19]. The resolution of this technique is determined by the frequency sweep laser and grating interval. Another technique is the optical time domain reflectometry (OTDR) system by employing a long FBG sensor and an ultra-short pulsed 
tunable laser, which has achieved distributed sensing with $1 \mathrm{~mm}$ spatial resolution [20]. To achieve such a high spatial resolution, both OFDR and OTDR system need very expensive light source, severely limiting their use in a wide range of applications [20].

In this paper, we propose and demonstrate for the first time a simple and low-cost distributed sensor with millimeter-order spatial resolution using a multichannel-FBG (MCFBG). The MC-FBG was designed using the layer peeling (LP) algorithm, in which each channel is spatially separated [21-24]. A 21-channel MC-FBG was fabricated using seamless UV-inscription with a uniform phase mask [25]. Real time temperature field mapping was demonstrated using a wavelength division multiplexing (WDM) technique to interrogate this device.

\section{Multichannel FBG design}

To achieve a high spatial resolution temperature field mapping, a multichannel grating needs to have a high density of sensing units. There are several methods to achieve this, including sampling gratings, Moiré grating structures, and using the layer peeling (LP) algorithm designed structures [26-28]. For sampled gratings, the channels are concentrated in the center of the grating and are not suitable for the point sensing or the distributed sensing [29]. Moiré grating designs lack of flexibility to design each channel of the grating independently [30]. In contrast, the layer peeling (LP) algorithm is a more ideal method to design a MC-FBG with the desired multichannel spectrum.

The target reflectivity of a MC-FBG can be expressed as [31],

$$
r(\lambda)=\sqrt{R} \times \sum_{j=1}^{N} \exp \left(-\left(\frac{2 \pi n_{e f f}}{a_{j}}\left(\frac{1}{\lambda}-\frac{1}{\lambda_{j}}\right)\right)^{b_{j}}\right) \exp \left(i 2 \pi n_{e f f}\left(\frac{1}{\lambda}-\frac{1}{\lambda_{B}}\right) d_{j}\right), \quad j=1,2,3 \ldots N(1)
$$

where $R, N, n_{\text {eff }}$ and $\lambda_{B}$ are the designed reflection, total channel number, effective refractive index and central wavelength of the full spectra, respectively; $\lambda_{j}, d_{j}$, and $a_{j}, b_{j}$ are the central wavelength, tailored group delay and the two parameters of super-Gaussian functions for channel $j$. To spatially separate the channels, we used staircase form tailored group delay $d_{j}$ for the grating design which is a function of the location of the channel given as

$$
d_{j}=2 \times\left[L_{0}+(j-1) L_{s}\right], \quad j=1,2,3 \ldots N
$$

where $L_{0}$ is location of the first channel, $L_{s}$ is spatial interval of channels.
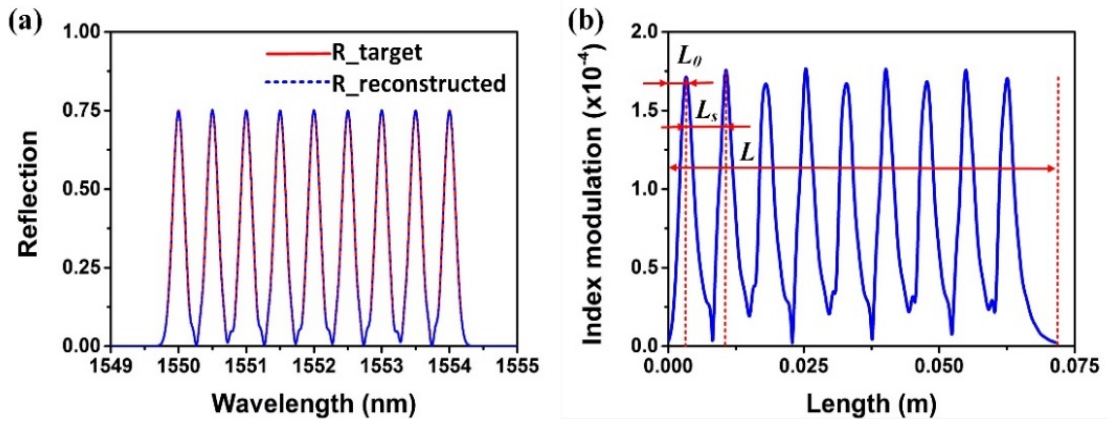

Fig. 1. (a) The target spectrum (red) and reconstructed spectrum (blue) of a 9-channel FBG. (b) The index modulation profile of the 9-channel FBG.

To demonstrate this approach, we synthesized a 9-channel design, in which the wavelength separation between each channel is $0.5 \mathrm{~nm}$, and $L_{0}, L_{s}$ are set as $3 \mathrm{~mm}, 7.4 \mathrm{~mm}$, respectively. We then reconstructed the spectrum from this design. The reconstructed spectrum is almost the same as the target spectrum, as shown in Fig. 1(a), in which the full 
width half maximum (FWHM) of the channel is $0.2 \mathrm{~nm}$ and the channel gap is $0.5 \mathrm{~nm}$. The index modulation profile of the 9-channel FBG clearly indicates that the 9 channels are evenly distributed along the grating, as illustrated in Fig. 1(b). The entire grating length $L$ is $71 \mathrm{~mm}$ and the spatial interval of the channels $L_{s}$ is $7.4 \mathrm{~mm}$ which is the spatial resolution of MC-FBG. It is clear that the channels are separated spatially along the grating due to the staircase group delay $d_{j}$.

The spatial distribution of one channel

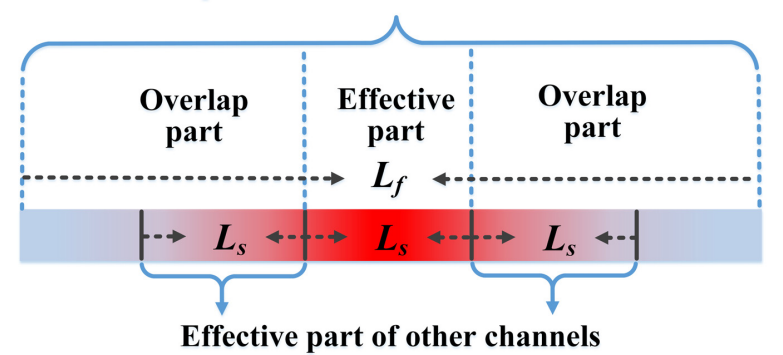

Fig. 2. The schematic of the channels overlap (the gradient of red represents the contribution to the channel spectrum).

In the MC-FBG structure, each channel is spatially distributed along the entire grating, thus all channels are overlapping with each other. However, the main effective part of each channel, which contributes to the major portion of the spectral shape, is much shorter than the entire grating. This is depicted in Fig. 2, where the gradient shown in red represents the spatial contribution of an individual channel to the channel spectrum. When used in a nonuniform temperature field, both the spectral interval and shape of the channel will change simultaneously due to the channel overlapping. The spatial interval and the channel gap will limit the maximum detectable temperature gradient, while, the spectral shape degradation decreases the signal noise ratio of the channel and affects the measurement accuracy.

(a)

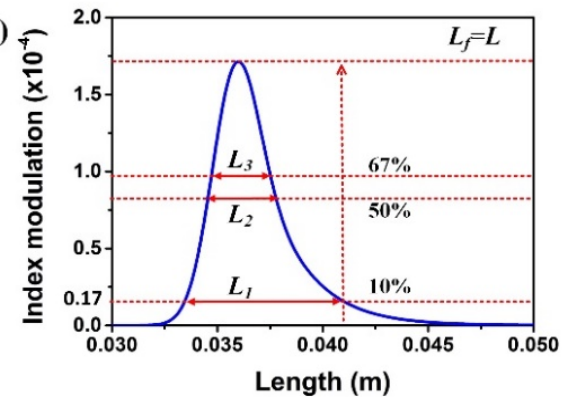

(b)

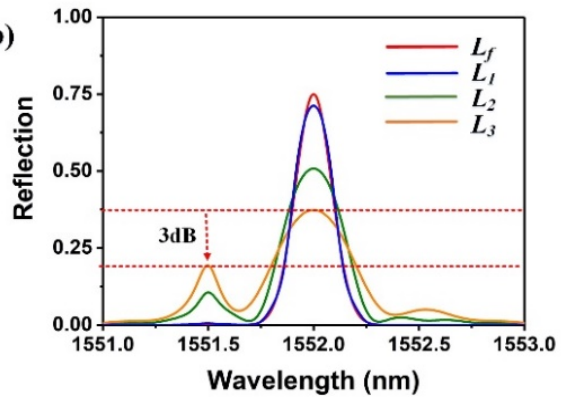

Fig. 3. (a) The index modulation profile of the channel (1-channel MC-FBG). (b) The spectral degradation under a non-uniform field ( $L_{f}$, full length, $L_{1}, L_{2}$, and $L_{3}$, are defined by the normal index modulation intensity of $10 \%, 50 \%$, and $67 \%$ ).

In the design, the spatial interval $L_{s}$ of the MC-FBG is a critical parameter, which is directly related to the sensing spatial resolution and the spectral shape degradation under a non-uniform temperature field. Because the MC-FBG is a continuously inscribed grating, the spatial interval of the MC-FBG is the effective length of each channel. To analyze and optimize the effective channel length $L_{s}$ of the MC-FBG, we reconstructed 1-channel MCFBG and its refractive index modulation profile is showed in Fig. 3(a). In this design, the full length $\left(\mathrm{L}_{\mathrm{f}}\right)$ of the channel is $71 \mathrm{~mm}$. To analyze the spectral shape degradation under a nonuniform temperature field, we chose three different effective channel lengths at three different index modulation intensity levels of $10 \%, 50 \%$, and $67 \%$ with respect to the maximum index 
modulation intensity, which were $L_{1}(2.7 \mathrm{~mm}), L_{2}(3.0 \mathrm{~mm})$ and $L_{3}(6.9 \mathrm{~mm})$ respectively, as shown in Fig. 3(a). Under a non-uniform temperature field, the MC-FBG is subjected to a temperature distribution along its length, which will cause the degradation of reflected spectra of the channel, resulting in more peaks appearing in the spectra, as shown in Fig. 3(b). From the figure, it can be clearly see that the grating with effective length $L_{l}$ showed very weak spectral degradation; as reducing the effective length, the visibility of spectra of the channel becomes worse, causing the reflected peak split into three peaks. The intensity ratio between side peak and main peak reaches to $3 \mathrm{~dB}$ as the effective channel length is $L_{3}$, which just matches the request of our interrogation system.

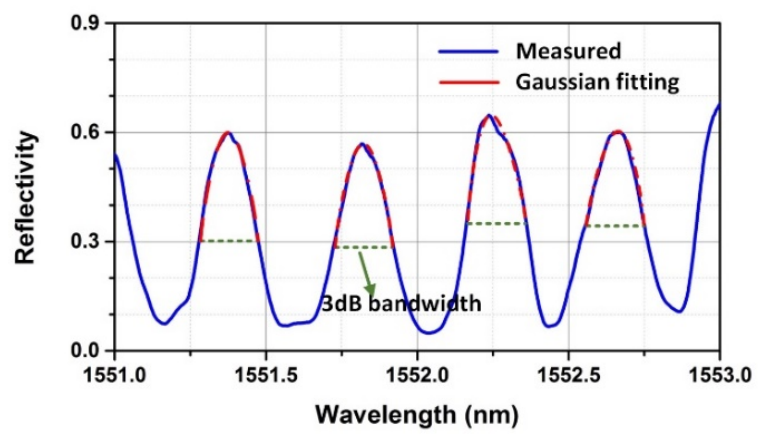

Fig. 4. The measured reflection spectrum using real-time interrogation method for the MCFBG.

The interrogation system was based on time domain spectral demodulation by monitoring the relative wavelength shift of each channel within $3 \mathrm{~dB}$ bandwidth, as shown in Fig. 4 . The target spectral profile of each channel was fitted by applying the super-Gaussian function to improve the stability and accuracy of the measurement, and the visibility of reflected spectrum of each channel should be larger than $3 \mathrm{~dB}$ to eliminate the influence of the spectral shape degradation. According to the previous analysis, we choose $3 \mathrm{~mm}$ as the effective channel length of the MC-FBG, and have designed a 21-channel FBG as shown in Fig. 5. The total grating length is $71 \mathrm{~mm}$ and the total bandwidth is $11 \mathrm{~nm}$.
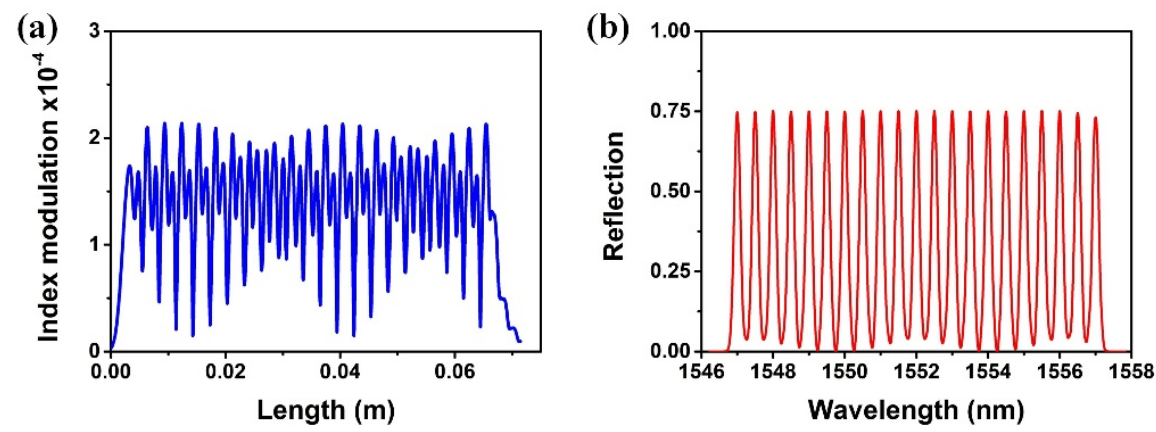

Fig. 5. (a) The index modulation profile. (b) The reconstructed spectrum of the 21-channel FBG.

\section{Grating fabrication}

A 21-channel FBG was fabricated using a seamless UV-inscription technique with a beam profile (BP) pre-compensation mechanism, as shown in Fig. 6(a) [25]. The inscription system uses a frequency doubled argon ion CW laser producing a $244 \mathrm{~nm}$ beam, which is modulated using an acoustic optical modulator (AOM) and collimated to a $270 \mu \mathrm{m}$ waist size to illuminate a $1071 \mathrm{~nm}$ period uniform phase mask (PM). A high precision air bearing linear 
stage is used to scan the fiber. The modulation of the laser beam is controlled so that successive exposure points overlay each other coherently. The position of the fiber is used to control the AOM to provide the required apodization and localized wavelength for the resulting FBG. The wavelength chirp can be achieved by a small phase offset between successive exposures. The grating was fabricated in standard telecommunications fiber (SMF 28) after hydrogenation for 2 days at 150 bar and $80^{\circ} \mathrm{C}$.

(a)

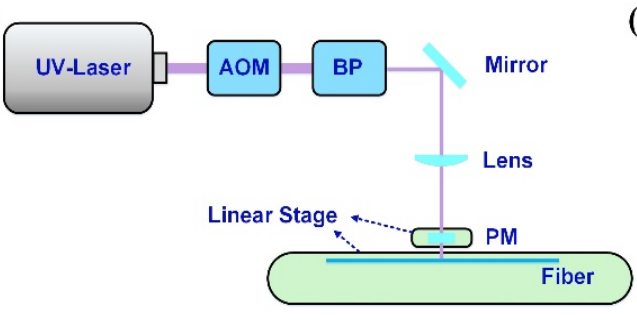

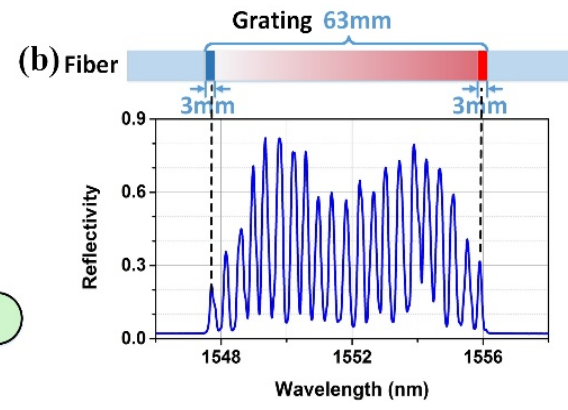

b) Fiber

Fig. 6. (a) The schematic of the fabrication system. (b) The structure and spectrum of the 21channel FBG.

After the grating inscription, the 21-channel FBG was annealed at $80{ }^{\circ} \mathrm{C}$ over 48 hours to stabilize its structure. Figure 6(b) shows the spectra of the MC-FBG, in which there are 21 channels with spectrum covering the wavelength range from $1547.76 \mathrm{~nm}$ to $1555.89 \mathrm{~nm}$. The total grating length is around $63 \mathrm{~mm}$, and the channel gap is $3 \mathrm{~mm}$. The FWHM of the individual channel spectrum is $0.2 \mathrm{~nm}$ and the channel wavelength separation is around 0.43 $\mathrm{nm}$.

\section{Temperature field monitoring}

The experimental setup used for the temperature field distribution measurement is shown in Fig. 7. An amplified spontaneous emission (ASE) light source emits broad-band light ranging from $1510 \mathrm{~nm}$ to $1590 \mathrm{~nm}$ through an optical circulator (OC) onto the 21-channel FBG; the spectrum of the reflected light is measured using a tunable FP filter (TFF) and photo detector (PD) and sampled by the analog to digital converter (ADC). The temperature gradient is generated using two thermal electronic cooler (TEC) stages and a metal plate, of which each end is fixed on to the TEC stage. The 21-channel FBG is held at the center area of the metal plate.

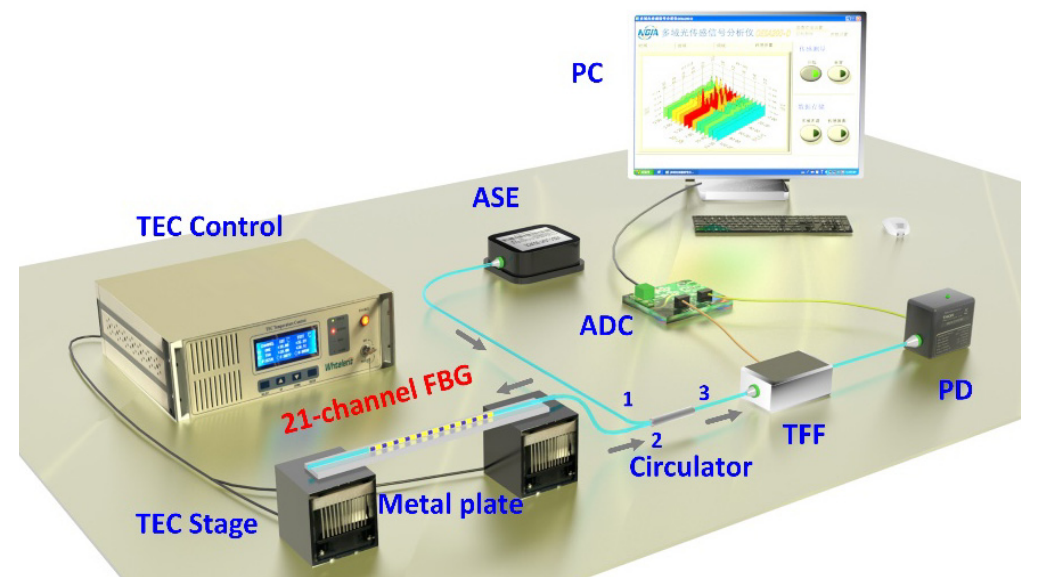

Fig. 7. Experimental setup for temperature field monitoring. 
The temperature sensitivity of the 21-channel FBG was calibrated by varying the temperature of the two TEC stages simultaneously from $5{ }^{\circ} \mathrm{C}$ to $80{ }^{\circ} \mathrm{C}$. The measured temperature responses of the 21 channels of the FBG are depicted in Fig. 8, where the curves with different colors represent the responses of different channels. The sensitivities of the channels varied from $9.428 \mathrm{pm} /{ }^{\circ} \mathrm{C}$ to $9.768 \mathrm{pm} /{ }^{\circ} \mathrm{C}$, which is similar to normal $\mathrm{FBGs}$, with all the adjusted R-square values $>0.999$. The maximum detectable temperature gradient of 7.85 ${ }^{\circ} \mathrm{C} / \mathrm{mm}$ is determined by the effective sensing bandwidth of $0.43 \mathrm{~nm}$ and the spatial resolution of $3 \mathrm{~mm}$.

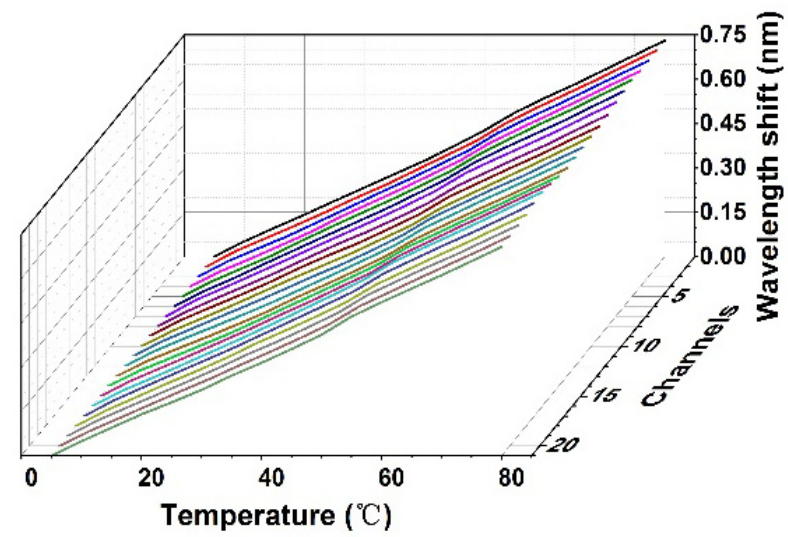

Fig. 8. The temperature sensitivity of the 21-channel FBG.

To produce a varying temperature field distribution in the experiment, the two TECs were set at different temperature levels. Initially, the two TEC stages were set to $25^{\circ} \mathrm{C}$, then over a time period of $175 \mathrm{~s}$ one had its temperature reduced to $10^{\circ} \mathrm{C}$ while the other was increased to $85{ }^{\circ} \mathrm{C}$ to produce a final temperature gradient of $1.00{ }^{\circ} \mathrm{C} / \mathrm{mm}$. The real time measured results are shown in Fig. 9(a), in which different curves correspond to different channels. The monitoring map depicts the temperature field distribution of the metal plate varying from uniform distribution to gradient distribution, in which we can see the temperature field became stable after $150 \mathrm{~s}$. Figure 9(b) shows the final temperature field distribution after 175 s. The temperature ranged from $23.32{ }^{\circ} \mathrm{C}$ to $80.46{ }^{\circ} \mathrm{C}$ giving a temperature gradient of 0.97 ${ }^{\circ} \mathrm{C} / \mathrm{mm}$, which is similar to the actual temperature gradient. The temperature response curve of the 21 th channel dropped at $50 \mathrm{~s}$ because the TEC stage took less time to reach $10^{\circ} \mathrm{C}$ than $85^{\circ} \mathrm{C}$. In addition, the slight non-linearity of the curve in Fig. 9(b) might be caused by the imperfect thermal conduction of the heat device.
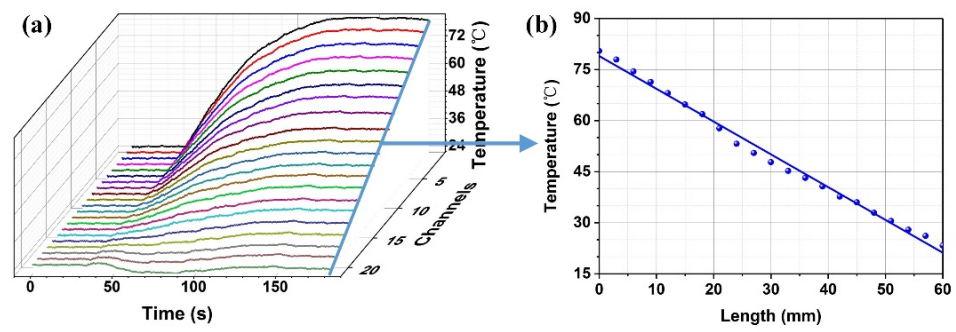

Fig. 9. (a) The real time monitoring of the temperature field variation (from uniform distribution to gradient distribution). (b) The temperature field monitored in the time of $175 \mathrm{~s}$ along the grating length.

Moreover, to test the performance of the sensing system in non-uniform gradient temperature field, the 21-channel FBG attached to the metal plate was heated up at the center 
position by a point heat source (an electric iron). The initial temperature of the metal plate was at the room temperature of $25{ }^{\circ} \mathrm{C}$ and the electric iron was set to $300{ }^{\circ} \mathrm{C}$. The whole temperature diffusing process was recorded using the MC-FBG based sensing system with the result shown in Fig. 10(a). At $38 \mathrm{~s}$, the iron touched the center of the metal plate and we can see the heat diffusing out and increasing the temperature of the surrounding region. At $116 \mathrm{~s}$, the iron was removed away and the metal plate quickly cooling down to the room temperature symmetrically. The measured response was compared to the calculated one using the heat transfer model shown in Fig. 10(b) simulated using the Comsol. Figure 10(c) shows the measured and simulated temperature profiles at $116 \mathrm{~s}$ by using proposed MC-FBG sensor, both the experimental and simulated results agreed well with each other, although there is a slight mismatch between the curves, which might be caused by misalignment between the fiber and the metal plate. The experimental results show again that the MC-FBG sensor is working well in non-uniform gradient temperature field.

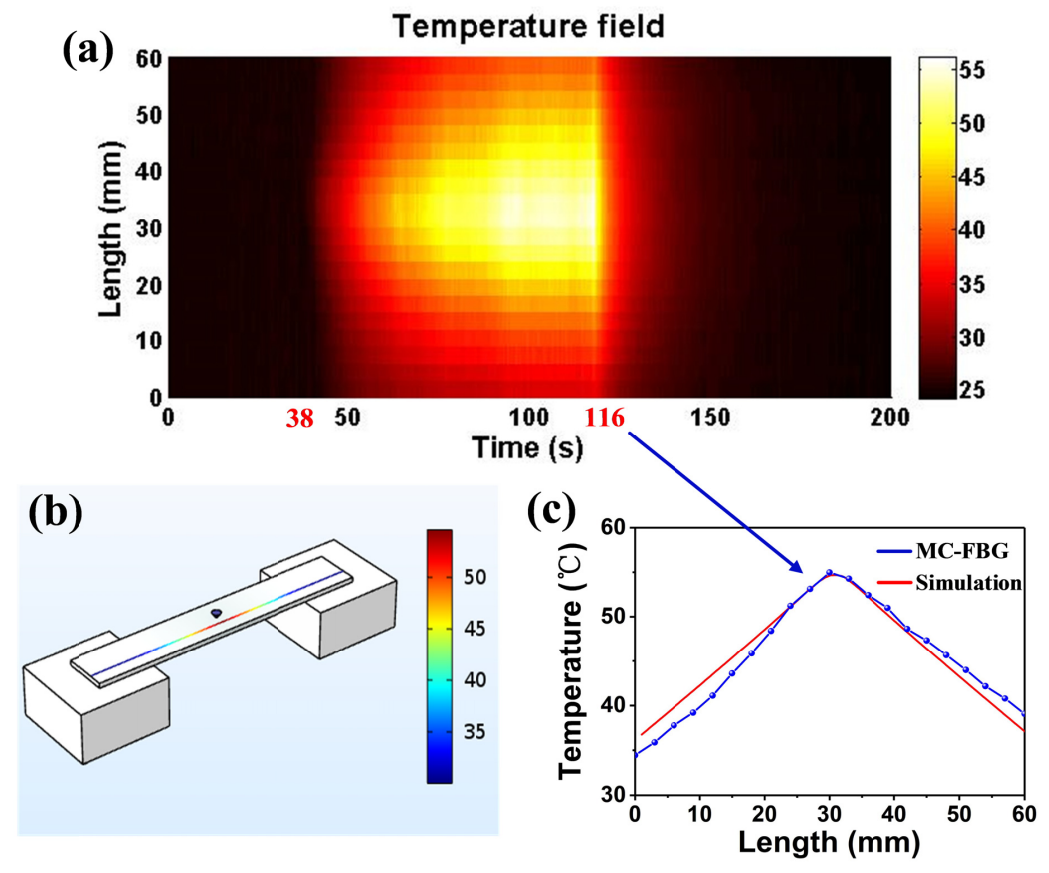

Fig. 10. (a) The temperature field map of the 21-channel FBG induced by the $300{ }^{\circ} \mathrm{C}$ point heat source. (b) The heat transfer model and the simulated temperature gradient at the position of fiber. (c) The comparison of the experimental and simulated temperature gradients at $116 \mathrm{~s}$.

\section{Conclusion}

In this work, we have demonstrated a real time temperature field sensor utilizing a 21-channel FBG. A tailored group delay profile is used to separate each channel of the grating spatially and the channel overlap is optimized to achieve a high spatial resolution. The channel gap of the sensor is $0.43 \mathrm{~nm}$ with a total spectrum bandwidth of $8.54 \mathrm{~nm}$. Real time monitoring of a temperature field is achieved experimentally with a resolution of $3 \mathrm{~mm}$, temperature sensitivity of $9.7 \mathrm{pm} /{ }^{\circ} \mathrm{C}$ and a maximum detectable temperature gradient of $7.85^{\circ} \mathrm{C} / \mathrm{mm}$. The achievable channel density is limited by the laser beam size used in current fabrication system $[25,31]$. Multiple MC-FBGs with different central wavelengths may be multiplexed in a single fiber to increase the sensing length and provide excellent potential for distributed sensing. In addition, the MC-FBG based sensing system is much simpler and cheaper than the OFDR or the ultra-short pulse OTDR schemes [17-20] for distributed sensing with 
millimeter-order spatial resolution. The MC-FBG sensor should be more suitable for practice temperature field monitoring applications.

\section{Funding}

National Natural Science Foundation of China (61775072, 61505244); Wuhan Morning Light Plan of Youth Science and Technology (2017050304010280); Science Fund for Creative Research Groups of the Nature Science Foundation of Hubei (2018CFA004); Major Projects of Technical Innovation of Hubei (2018AAA040).

\section{References}

1. N. N. Jarenwattananon, S. Glöggler, T. Otto, A. Melkonian, W. Morris, S. R. Burt, O. M. Yaghi, and L. S. Bouchard, "Thermal maps of gases in heterogeneous reactions," Nature 502(7472), 537-540 (2013).

2. M. Mecklenburg, W. A. Hubbard, E. R. White, R. Dhall, S. B. Cronin, S. Aloni, and B. C. Regan, "Nanoscale temperature mapping in operating microelectronic devices," Science 347(6222), 629-632 (2015).

3. H. Li, H. Yang, E. Li, Z. Liu, and K. Wei, "Wearable sensors in intelligent clothing for measuring human body temperature based on optical fiber Bragg grating," Opt. Express 20(11), 11740-11752 (2012).

4. X. Wu and R. Hull, "A novel nano-scale non-contact temperature measurement technique for crystalline materials," Nanotechnology 23(46), 465707 (2012).

5. X. Bao and L. Chen, "Recent progress in distributed fiber optic sensors," Sensors (Basel) 12(7), 8601-8639 (2012).

6. V. Márquez-Cruz and J. Albert, "High resolution NIR TFBG-assisted biochemical sensors," J. Lightwave Technol. 33(16), 3363-3373 (2015).

7. J. W. Arkwright, N. G. Blenman, I. D. Underhill, S. A. Maunder, M. M. Szczesniak, P. G. Dinning, and I. J. Cook, "In-vivo demonstration of a high resolution optical fiber manometry catheter for diagnosis of gastrointestinal motility disorders," Opt. Express 17(6), 4500-4508 (2009).

8. R. Rathod, R. D. Pechstedt, D. A. Jackson, and D. J. Webb, "Distributed temperature-change sensor based on Rayleigh backscattering in an optical fiber," Opt. Lett. 19(8), 593-595 (1994).

9. J. Song, W. Li, P. Lu, Y. Xu, L. Chen, and X. Bao, "Long-range high spatial resolution distributed temperature and strain sensing based on optical frequency-domain reflectometry," Photonics J. 6(3), 1-8 (2014).

10. M. A. Soto, P. K. Sahu, S. Faralli, G. Bolognini, F. Di Pasquale, B. Nebendahl, and C. Rueck, "Distributed temperature sensor system based on Raman scattering using correlation-codes," Electron. Lett. 43(16), 862-864 (2007).

11. M. A. Soto, T. Nannipieri, A. Signorini, A. Lazzeri, F. Baronti, R. Roncella, G. Bolognini, and F. Di Pasquale, "Raman-based distributed temperature sensor with $1 \mathrm{~m}$ spatial resolution over $26 \mathrm{~km}$ SMF using low-repetitionrate cyclic pulse coding," Opt. Lett. 36(13), 2557-2559 (2011).

12. K. Y. Song, Z. He, and K. Hotate, "Distributed strain measurement with millimeter-order spatial resolution based on Brillouin optical correlation domain analysis," Opt. Lett. 31(17), 2526-2528 (2006).

13. A. Masoudi, M. Belal, and T. P. Newson, "Distributed dynamic large strain optical fiber sensor based on the detection of spontaneous Brillouin scattering," Opt. Lett. 38(17), 3312-3315 (2013).

14. M. A. Soto, J. A. Ramírez, and L. Thévenaz, "Intensifying the response of distributed optical fibre sensors using 2D and 3D image restoration," Nat. Commun. 7(1), 10870 (2016).

15. D. A. Kersey, M. A. Davis, H. J. Patrick, M. LeBlanc, K. P. Koo, C. G. Askins, M. A. Putnam, and E. Joseph Friebele, "Fiber grating sensors," J. Lightwave Technol. 15(8), 1442-1463 (1997).

16. J. W. Arkwright, I. D. Underhill, S. A. Maunder, N. Blenman, M. M. Szczesniak, L. Wiklendt, I. J. Cook, D. Z. Lubowski, and P. G. Dinning, "Design of a high-sensor count fibre optic manometry catheter for in-vivo colonic diagnostics," Opt. Express 17(25), 22423-22431 (2009).

17. K. Yuksel, V. Moeyaert, P. Megret, and M. Wuilpart, "Complete analysis of multireflection and spectralshadowing crosstalks in a quasi-distributed fiber sensor interrogated by OFDR," IEEE Sens. J. 12(5), 988-995 (2012).

18. A. K. Sang, M. E. Froggatt, S. T. Kreger, and D. K. Gifford, "Millimeter resolution distributed dynamic strain measurements using optical frequency domain reflectometry," Proc. SPIE 7753, 77532S (2011).

19. D. Wada, H. Igawa, and T. Kasai, "Vibration monitoring of a helicopter blade model using the optical fiber distributed strain sensing technique," Appl. Opt. 55(25), 6953-6959 (2016).

20. A. L. Ricchiuti, D. Barrera, K. Nonaka, and S. Sales, "Temperature gradient sensor based on a long-fiber Bragg grating and time-frequency analysis," Opt. Lett. 39(19), 5729-5731 (2014).

21. R. Feced, M. N. Zervas, and M. A. Muriel, "An efficient inverse scattering algorithm for the design of nonuniform fiber Bragg gratings,” IEEE J. Quantum Electron. 35(8), 1105-1115 (1999).

22. J. Skaar, L. Wang, and T. Erdogan, "On the synthesis of fiber Bragg gratings by layer peeling," IEEE J. Quantum Electron. 37(2), 165-173 (2001).

23. J. Skaar and O. H. Waagaard, "Design and characterization of finite-length fiber gratings," IEEE J. Quantum Electron. 39(10), 1238-1245 (2003).

24. H. Li and Y. Sheng, "Direct design of multichannel fiber Bragg grating with discrete layer-peeling algorithm," IEEE Photonic. Tech. L. 15(9), 1252-1254 (2003). 
25. E. G. Turitsyna, A. Gbadebo, and J. A. Williams, "A technique for mitigating the effect of the writing-beam profile on fibre Bragg grating fabrication," Opt. Express 23(10), 12628-12635 (2015).

26. M. Li, X. Chen, J. Hayashi, and H. Li, "Advanced design of the ultrahigh-channel-count fiber Bragg grating based on the double sampling method," Opt. Express 17(10), 8382-8394 (2009).

27. L. A. Everall, K. Sugden, J. A. R. Williams, I. Bennion, X. Liu, J. S. Aitchison, S. Thoms, and R. M. De La Rue, "Fabrication of multipassband moiré resonators in fibers by the dual-phase-mask exposure method," Opt. Lett. 22(19), 1473-1475 (1997)

28. A. Lin, L. Wang, W. Zhao, X. Hu, X. Liu, and Y. Gong, "Optimal design of multichannel fiber Bragg grating filters with small dispersion and low index modulation," J. Lightw. Tech. 27(15), 3235-3240 (2009).

29. M. Li and J. Yao, "Multichannel arbitrary-order photonic temporal differentiator for wavelength-divisionmultiplexed signal processing using a single fiber Bragg grating," J. Lightw. Tech. 29(17), 2506-2511 (2011).

30. A. M. Gillooly, H. Dobb, L. Zhang, and I. Bennion, "Distributed load sensor by use of a chirped moiré fiber Bragg grating," Appl. Opt. 43(35), 6454-6457 (2004).

31. H. Cao, J. Atai, X. Shu, and G. Chen, "Direct design of high channel-count fiber Bragg grating filters with low index modulation," Opt. Express 20(11), 12095-12110 (2012). 